EindV auch sonst keine Regelungen zur Zweckbindung der Datenverwendung. Eine damit von der Verordnung in Kauf genommene Preisgabe sensibler Gesundheitsdaten des vorgenannten Ausmaßes stellt einen gravierenden Eingriff in die Rechte des ASt. dar.

Der AG. hat nicht dargelegt, dass bei Erfolg des Antrags auf einstweiligen Rechtsschutz vergleichbare schwere Folgen in Rede stehen. Der Erlass der einstweiligen Anordnung hat nicht zur Folge, dass die Nachweispflicht für Personen, denen die Verwendung einer Mund-Nasen-Bedeckung wegen einer Behinderung oder aus gesundheitlichen Gründen nicht möglich oder unzumutbar ist, gänzlich entfällt. Vielmehr ist die Befreiung aus gesundheitlichen Gründen vor Ort - wie bis zum Erlass der 3. SARS-CoV-2-EindV - durch ein ärztliches Zeugnis, das Namen und Geburtsdatum enthält, d.h. eine Identifikation erlaubt, nachzuweisen. Soweit der AG. geltend macht, er habe die Anforderungen an das ärztliche Zeugnis präzisiert, um der Gefahr von Gefälligkeitsattesten ,wirksam vorzubeugen“, hat er diese Gefahr bereits nicht hinreichend belegt. Dabei ist zu berücksichtigen, dass die Maskenpflicht nur dann unterlaufen zu werden droht, wenn Gefälligkeitsatteste in nennenswertem Umfang im Raume stünden. Hierfür führt der AG. indes nichts an. Vielmehr verweist er zu Recht selbst darauf, das das Ausstellen unrichtiger Gesundheitszeugnisse nach $\$ 278$ $\mathrm{StGB}$ strafbewehrt ist. Soweit er auf verwaltungsgerichtliche Rechtsprechung (VG Würzburg, Beschl. v. 16.9.2020 - W 8 E 20.1301 -, juris, Rdnr. 22; VG Düsseldorf, Beschl. v. 25. 8. 2020 - 18 L 1608/20 -, juris, Rdnr. 37; BayVGH, Beschl. v. 26.10.2020 - 20 CE 20.2185 -, juris, Rdnr. 19) Bezug nimmt, betrifft diese bereits nicht die (aktuelle) Situation im Land Brandenburg. Unabhängig hiervon ist diese Rechtsprechung zu Anträgen auf Feststellung, dass eine Befreiung von der Maskenpflicht aus gesundheitlichen Gründen im Einzelfall besteht, ergangen. In diesen Fällen war die Prüfung jeweils nicht von privaten Stellen, sondern von einem ohnehin datenschutzrechtlichen Bindungen unterliegenden Gericht vorgenommen worden. Soweit darin im Einzelfall festgestellt wird, dass das Gericht ein ärztliches Attest als Nachweis für eine Befreiung aus gesundheitlichen Gründen nur dann für ausreichend überprüfbar hält, wenn dieses auch eine medizinische Diagnose und die sich hieraus ergebenden Folgen für das Tragen einer Mund-Nasen-Bedeckung beschreibt, bedeutet dies nicht zwangsläufig, dass eine wirksame Kontrolle des Befreiungstatbestandes die Vorlage eines solchen Attestes „,vor Ort“ überall dort, wo eine Maskenpflicht gilt, erfordert.

https://doi.org/10.1007/s00350-021-6068-7

\section{Anmerkung zu OVG Berlin-Brandenburg, Beschl. v. 4.1.2021 - OVG 11 S 132/20:}

\section{Bernd Klemp}

Dass die Diagnose eine entscheidende Rolle für die Freistellung von der Pflicht zum Tragen einer Mund-NasenBedeckung spielt, steht außer Frage. Dass diese jedoch nicht in allen Situationen in dem Originalattest preisgegeben werden müsste, hat das Gericht richtigerweise erkannt.

Um Diskriminierungen zu vermeiden und das allgemeine Persönlichkeitsrecht zu schützen, ist es angemessen im öffentlichen Raum - u.a. bei alltäglichen Einkaufssituationen, in der Apotheke, in der Poststelle oder in öffentli-

Rechtsanwalt Bernd Klemp, LL.M.

Fachanwalt für Medizinrecht und Fachanwalt für Versicherungsrecht, Bietmann Rechtsanwälte Steuerberater PartmbB,

Martinstraße 22-24, 50667 Köln, Deutschland chen Verkehrsmitteln - auf die Offenbarung der Diagnose zu verzichten. Zum gegenwärtigen Zeitpunkt der Pandemie wird die Glaubhaftmachung der Freistellungssituation durch Nichtangabe der Diagnose im Originalattest nicht erschüttert, da es keine Anhaltspunkte gibt, dass Gefälligkeitsatteste von Ärzten ausgestellt würden.

Vergleichbar zu anderen Entscheidungen ${ }^{1}$, wo innerhalb des Schulbetriebs die Diagnose im Attest enthalten sein sollte, besteht dort nicht die Gefahr, dass datenschutzwidriger Umgang gegeben sei. Denn das Attest wird in einem geschützten Rahmen vorgezeigt, wo interne Vorschriften den Umgang von persönlichen Daten gewährleisten.

Daher wird es angemessen sein anhand Einzelfallbetrachtungen zu entscheiden, wann die Diagnose im Attest enthalten sein sollte und wann nicht. Im öffentlichen Raum, wo der datenschutzkonforme Umgang nicht gewährleistet werden kann, überwiegt der Schutz des allgemeinen Persönlichkeitsrechts des Freigestellten.

1) Z.B. OVG Münster, Beschl. v. 24.9.2020 - 13 B 1368/20.

\section{Voraussetzungen einer Sonderbedarfsfeststellung}

GG Art. 12 Abs. 1; SGB V §§95 Abs. 2, 101 Abs. 1 S. 1 Nr. 3 , 285 Abs. 1, Abs. 3 S. 1; BedarfsplanungsRL §§36, 37; DSGVO Art. 6 Abs. 1 und 2

1. In einem Rechtsstreit über eine Sonderbedarfsanstellung ist die Beiladung des anzustellenden Arztes nicht erforderlich.

2. Bei der Entscheidung über eine Sonderbedarfszulassung oder -anstellung sind die Grundsätze über Vornahmeklagen anzuwenden. Danach sind grundsätzlich alle Tatsachenänderungen bis zur mündlichen Verhandlung der letzten Tatsacheninstanz und alle Rechtsänderungen bis zum Abschluss der Revisionsinstanz zu berücksichtigen.

3. Auch eine Zusatzweiterbildung oder eine $\mathrm{Zu}$ satzbezeichnung kann einen qualifikationsbezogenen Sonderbedarf begründen, wenn sie den in $\$ 37$ Abs. 1 BedarfsplanungsRL genannten Qualifikationen vom zeitlichen und qualitativen Umfang her gleichsteht.

4. Bei der Bedarfsprüfung sind nur reale, nicht dagegen potenzielle Versorgungsangebote zu berücksichtigen, die tatsächlich nicht zur Verfügung stehen, weil Leistungserbringer nicht zur Erbringung weiterer Leistungen bereit oder tatsächlich nicht in der Lage sind.

5. Bei der Feststellung von Sonderbedarf haben die Zulassungsgremien gemä $\$ 36$ Abs. 3 Nr. 1 BedarfsplanungsRL zur Feststellung einer unzureichenden Versorgungslage zunächst eine Region abzugrenzen, die vom beantragten Ort der Niederlassung aus versorgt werden soll und die dortige Versorgungslage zu bewerten.

6. Der Versorgungsbedarf am beantragten Ort allein begründet noch keinen qualifikationsbezogenen Sonderbedarf. Entscheidend ist, ob dieser nicht durch andere (zumutbar erreichbare) Praxen gedeckt werden kann, auch wenn dies bedeutet, dass die Versicherten nicht an ihrem Wohnort oder in unmittelbarer Nähe ihres Wohnortes behandelt werden.

7. Ohne Bedeutung ist, ob sich die Patienten die Behandlung gerade an einem bestimmten Ort oder beson-

\section{Eingesandt und bearbeitet}

von Rechtsanwalt und Notar Dr. iur. Paul Harneit,

Fachanwalt für Medizinrecht, CausaConcilio Rechtsanwälte, Deliusstraße 16, 24114 Kiel, Deutschland 